\title{
An Advanced Broyden-Fletcher-Goldfarb-Shanno Algorithm for Prediction and Output-Related Fault Monitoring in Case of Outliers
}

\author{
Cuiping Xue $\mathbb{D}^{1,2}$ Tie Zhang $\mathbb{D}^{1},{ }^{1}$ and Dong Xiao $\mathbb{B D}^{2,3}$ \\ ${ }^{1}$ College of Science, Northeastern University, Shenyang 110819, China \\ ${ }^{2}$ Engineering and Liaoning Key Laboratory of Intelligent Diagnosis and Safety for Metallurgical Industry, \\ Northeastern University, Shenyang 110819, China \\ ${ }^{3}$ College of Information Science, Northeastern University, Shenyang 110819, China
}

Correspondence should be addressed to Cuiping Xue; xuecuiping@mail.neu.edu.cn

Received 26 November 2021; Revised 4 January 2022; Accepted 15 January 2022; Published 4 February 2022

Academic Editor: Jose Corchado

Copyright (C) 2022 Cuiping Xue et al. This is an open access article distributed under the Creative Commons Attribution License, which permits unrestricted use, distribution, and reproduction in any medium, provided the original work is properly cited.

In the process industry, fault prediction and product-related fault monitoring are important links to ensure product quality and improve economic benefits. In this paper, under the framework of the BFGS (Broyden-Fletcher-Goldfarb-Shanno) algorithm, a new and more accurate data-driven method, the ABFGS algorithm, is proposed. Compared with the BFGS algorithm, the ABFGS algorithm adds output-related fault monitoring capabilities and has strong robustness, which can eliminate the influence of outliers on measurement data. The effectiveness of this method has been verified by the Eastman benchmark program in Tennessee. The simulation results show that this method can eliminate the influence of outliers and effectively predict the process. Compared with the other three algorithms, the ABFGS algorithm can not only clearly and accurately indicate whether the detected fault is related to the output but also provide a higher fault monitoring rate.

\section{Introduction}

Industrial production of a set of large equipment is composed of a large number of working parts, connected to each other, and between different parts together, a component failure often causes a chain reaction; if some small faults cannot be ruled out in time, this will lead to the whole system paralysis or even the whole manufacturing process, light influence production, or downtime production; even the machine is destroyed, causing casualties. Therefore, as an important technology to improve process safety and ensure product quality, process monitoring has been highly valued by academia and industry in recent twenty years $[1,2]$. Common methods are the model-based method [3] and the data-driven method [4]. Data-driven technology requires less prior knowledge, has no accurate system model, only depends on historical process measurement, and is more applicable to output-related monitoring. The most studied data-driven methods are principal component analysis (PCA) [5] and partial least squares (PLS).

In the past, the quality of output products was estimated by the experience of skilled technicians, which brought potential risks to the safety and reliability of the process. In order to ensure that the output products meet the standards, prediction [6] and output-related fault monitoring has become an active field. BFGS (Broyden-Fletcher-Goldfarb-Shanno) algorithm is introduced in this paper to monitor output-related faults. BFGS algorithm is a quasi-Newton algorithm [7-10] independently proposed by Broyden, Fletcher, Goldfarb, and Shanno in 1970 to solve unconstrained optimization problems. BFGS algorithm and PLS algorithm belong to the conjugate direction method of optimization. BFGS algorithm not only satisfies the property of conjugate direction but also is the quasi-Newton algorithm with the best numerical stability so far. BFGS algorithm has the advantages of conjugate direction method and quasi-Newton method [11]: fast convergence and little iteration. BFGS 
algorithm is an efficient method for processing large amounts of data for quality prediction and fault monitoring [12]. It decomposes the input data space into main subspace and residual subspace, which are usually monitored by Hotelling's statistics and the SPE statistics, respectively. Because the BFGS algorithm decomposes the available data space obliquely, the output-related process variables may be included in the residual space, so two commonly used statistical data become unsuitable for output-related fault monitoring [13].

The measured values collected from industrial processes usually contain outliers. Statistically speaking, outliers are samples with different characteristics from most data. The method of studying the problem of inclusion outliers needs strong anti-interference ability [14-17]. Generally speaking, outliers can be divided into two types, namely, (1) highly leveraged data, which is far away from the data center in the available space, and (2) highly residual data, where there is a large deviation between the actual value and the predicted value in the product quality variable space [18]. Outliers may occur for several reasons; for example, the measurement process may be incorrectly observed and recorded or copied to a historical database. The presence of outliers can have a serious adverse effect on modeling and may distort the estimation of parameters, resulting in poor performance of the model $[19,20]$. Usually, outliers will affect the normal distribution of data sets and bring difficulties to the BFGS algorithm based on the assumption of normal distribution process measurement. Therefore, outliers are paid more and more attention to in data preprocessing and data modeling. Some data modeling methods reduce the influence of outliers by detecting and eliminating outliers during data preprocessing [21-23]. While eliminating outliers can be effective in some processes, caution is needed because the elimination process can also erase important hidden information, which can have a negative impact later in the data modeling process, for example, lack of robustness to high leverage and high residual.

In this paper, we propose an output-related prediction and fault monitoring algorithm called Advanced BFGS (ABFGS) algorithm. This method improves the BFGS algorithm in two aspects. (1) In order to achieve more accurate process prediction, the weight of outliers can be reduced with less calculation by selecting an appropriate weighting scheme. (2) By orthogonal decomposition, the output-related and output-unrelated subspaces are established, and more effective monitoring statistics are developed for the monitoring scheme. Therefore, the ABFGS algorithm can not only eliminate the influence of outliers on the regression coefficient but also avoid the problem of losing important information. Finally, the validity of the ABFGS algorithm is verified by Tennessee Eastman (TE) benchmark process.

This paper is organized as follows. In the second section, the BFGS algorithm and existing problems are discussed. In the third section, the output-related prediction and fault monitoring of ABFGS are proposed. The fourth section reports the simulation results of the TE process using an industrial benchmark. Finally, the fifth part summarizes the thesis.
Notation: $\mathbf{g}_{k}=\mathbf{g}\left(\mathbf{B}_{k}\right)=\nabla f\left(\mathbf{B}_{k}\right)$ represents the gradient, which is a vector composed of all partial derivatives $\left(\partial f(\mathbf{B}) / \partial \mathbf{B}_{i}\right)$ as components, where $\mathbf{B}_{i}$ is the $i$ th component of vector $\mathbf{B}$. $\|\mathbf{a}\|$ represents the second norm of vector $\mathbf{a}$, that is, the sum of squares of each component of $\mathbf{a}$, and then the root sign is opened. $F_{l, n-l, \alpha}$ represents the critical value of the right tail probability $\alpha$ of $F$ distribution with degrees of freedom of $l$ and $n-l$ in statistics. $\chi_{h, \alpha}^{2}$ represents the critical value of the right tail probability $\alpha$ of $\chi^{2}$ distribution with degree of freedom of $h$ in statistics. $\left|x_{i}-x_{j}\right|$ represents the absolute value of the difference between two numerical values. $[n / 2]$ is the integer sign, and the largest integer not larger than $n / 2$ is obtained.

\section{Preparing Knowledge and Problem Expression}

2.1. Process Prediction and Fault Monitoring Based on BFGS Algorithm. Given a normalized input data matrix $\mathbf{X} \in \mathbf{R}^{N \times m}$ and the corresponding normalized output matrix $\mathbf{Y} \in \mathbf{R}^{N \times 1}$, where $N$ is the number of sample points, $m$ is the number of process variables. The objective function is

$$
\begin{aligned}
\min f(\mathbf{B}) & =\frac{1}{2}\|\mathbf{Y}-\mathbf{X B}\|^{2} \\
& =\frac{1}{2}\left(\mathbf{Y}^{T} \mathbf{Y}+\mathbf{B}^{T} \mathbf{X}^{T} \mathbf{X B}-2 \mathbf{Y}^{T} \mathbf{X B}\right) .
\end{aligned}
$$

The gradient of the objective function $f(\mathbf{B})$ is

$$
\nabla f(\mathbf{B})=\mathbf{X}^{T} \mathbf{X B}-\mathbf{X}^{T} \mathbf{Y} .
$$

For the convenience of writing, remember $\mathbf{g}_{k}=\mathbf{g}\left(\mathbf{B}_{k}\right)=\nabla f\left(\mathbf{B}_{k}\right)=\mathbf{X}^{T} \mathbf{X B}_{k}-\mathbf{X}^{T} \mathbf{Y}$. The specific steps of BFGS Algorithm 1 in fault detection are given in the following [12].

The regression coefficient $\mathbf{M}=\mathbf{P b}$ of output $\mathbf{Y}$ to input $\mathbf{X}$ can be obtained by the BFGS algorithm. For a new normalized sample $\mathbf{x}_{\text {new }} \in \mathbf{R}^{1 \times m}$, its corresponding predicted output is $\bar{y}=\mathbf{x}_{\text {new }} \mathbf{M}$. In addition, the BFGS algorithm divides the input space into main subspace and residual space, namely, $\mathbf{X}=\widehat{\mathbf{X}}+\widetilde{\mathbf{X}}=\mathbf{T} \mathbf{P}^{T}+\widetilde{\mathbf{X}}$. The fault monitoring of two spaces adopts statistics $T^{2}$ and SPE, respectively. The calculation formula is as follows:

$$
\begin{aligned}
T^{2} & =\mathbf{t}^{T}\left(\frac{\mathbf{T}^{T} \mathbf{T}}{n-1}\right)^{-1} \mathbf{t}, \\
\mathrm{SPE} & =\mathbf{X}^{T}\left(\mathbf{I}-\mathbf{P} \mathbf{P}^{T}\right) \mathbf{X} .
\end{aligned}
$$

The calculation formula of two statistics of new sample points is

$$
\begin{aligned}
\mathbf{t}_{\text {new }}^{2} & =\mathbf{x}_{\text {new }} \mathbf{P} \Lambda^{-1} \mathbf{P}^{T} \mathbf{x}_{\text {new }}^{T}, \text { where } \boldsymbol{\Lambda}=\frac{1}{n-1} \mathbf{T}^{T} \mathbf{T}=\frac{1}{n-1} \mathbf{P}^{T} \mathbf{X}^{T} \mathbf{X P}, \\
\text { spe }_{\text {new }} & =\left\|\mathbf{x}_{\text {new }}\left(I-\mathbf{P} \mathbf{P}^{T}\right)\right\|^{2} .
\end{aligned}
$$


When the significance level $\alpha$ is given, the threshold value of statistical information is given by the following formula:

$$
J_{t h, T^{2}}=\frac{l\left(n^{2}-1\right)}{n(n-1)} F_{l, n-l, \alpha} .
$$

Here, $n$ is the number of sampled samples and $l$ is the number of reserved principal components.

$$
J_{t h, \mathrm{SPE}}=g \chi_{h, \alpha}^{2} .
$$

Here, $g=\rho^{2} / 2 \mu, h=2 \mu^{2} / \rho^{2}$, and $\mu$ and $\rho^{2}$ are the mean and variance of SPE statistics of the sample. When $t_{\text {new }}^{2}>J_{t h, T^{2}}$, the main subspace fault exists; when spe $_{\text {new }}>J_{t h, \mathrm{SPE}}$, the residual subspace fault exists.

2.2. Problems in the BFGS Algorithm. Running the BFGS algorithm on the input space is oblique decomposition for the output $\mathbf{Y}$. The main part $\widehat{\mathbf{X}}$ contains the orthogonal information of $\mathbf{Y}$, and the residual part $\widehat{\mathbf{X}}$ contains the related information of $\mathbf{Y}$. Therefore, the BFGS algorithm cannot be well used for output-related fault monitoring. In addition, the measured data collected from actual industrial processes usually contain some outliers, which may affect the normal distribution of data. However, the BFGS algorithm is sensitive to these outliers. Specifically, the obtained principal components cannot accurately reflect the characteristics of most data, so satisfactory monitoring results cannot be obtained.

\section{Advanced BFGS Algorithm}

3.1. Robust Normalization. Multivariate process measurement should be normalized in analysis. When the data set does not contain outliers, the normalization uses the following formula:

$$
\begin{aligned}
& \bar{x}=\frac{1}{n} \sum_{i=1}^{n} x_{i}, \\
& s^{2}=\frac{1}{n-1} \sum_{i=1}^{n}\left(x_{i}-\bar{x}\right)^{2} .
\end{aligned}
$$

Mean $\bar{x}$ and variance $s^{2}$ can show the center and divergence of measurement, respectively. However, when outliers appear in the data, especially when the number of outliers is large, the mean and variance may seriously deviate from the actual values. These deviations will lead to inaccurate prediction results and loss of application value.

When the data set contains outliers, robust normalization can be used. In this paper, the weighted center of sample points is defined as

$$
\bar{e}=\frac{\left(\sum_{i=1}^{n} w_{i} x_{i}\right)}{\sum_{i=1}^{i=1} w_{i}} .
$$

The weight coefficient formula is $w_{i}=(1 / 1+$ $\left.\left\|x_{i}-\widehat{x}\right\|^{2 * m}\right)$. Here, $\hat{x}$ is the median of all sample points, and $m \in N *$ is a constant. Its size can be trained by training sample points. The variance formula is

$$
Q_{n}=\eta\left\{\left|x_{i}-x_{j}\right| \mid i<j\right\}_{(k)} .
$$

Here, $k=\left(\begin{array}{l}h \\ 2\end{array}\right)=h(h-1) / 2, h=[n / 2]+1$, and [] represents the integer part. Under the condition $i<j$, we calculate $\left|x_{i}-x_{j}\right|$. Then, the obtained data are sorted in ascending order. Multiply the $k$ th by $\eta$ getting variance $Q_{n}$, where $\eta=2.2219$ is the correction coefficient [24]. Next, the original data can be normalized by weighted center $\bar{e}$ and variance $Q_{n}$.

3.2. Prediction Based on ABFGS Algorithm. ABFGS algorithm is a robust version of the BFGS algorithm, which reduces the influence of outliers by choosing an appropriate weighting scheme. Outliers refer to points far from the record center. In the ABFGS algorithm, their influence will be limited by a smaller weight coefficient. In the input variable space and the output variable space, there are two kinds of outliers: high leverage point and high residual point $[18,25]$, respectively. In the ABFGS algorithm, the formulas of two weights: leverage weight $w_{i}^{x}$ and residual weight $w_{i}^{r}$ are as follows:

$$
\begin{aligned}
& w_{i}^{x}=f\left(\frac{l_{i}}{\bar{e}_{i}\left(l_{i}\right)}, c\right), \\
& w_{i}^{r}=f\left(\frac{r_{i}-\bar{e}(r)}{\bar{e}_{i}\left(\left|r_{i}-\bar{e}(r)\right|\right)}, c\right) .
\end{aligned}
$$

Here, $f(z, c)=\left(1 / 1+(z / c)^{2}\right)$, the weight function $f(\cdot, \cdot)$ is the Fair function, $z$ is a variable, $c$ is a tuning constant, which is usually set as $c=4$, and $\bar{e}(\cdot)$ is calculated by the weight center of this paper, which can be calculated by formula (9). In this paper, $l_{i}$ is calculated by formula $l_{i}=\left\|x_{i}-\bar{e}(\mathbf{X})\right\|^{2} . x_{i}$ is the point on the convex set $\mathbf{X}$, definition $r_{i}=y_{i}-\mathbf{t}^{i} \mathbf{b}$, where $r_{i}$ represents the difference between the $i$ th observed value and the predicted value, $\mathbf{t}^{i}$ is the $i$ th row of the score matrix $\mathbf{T}$, and $\mathbf{b}$ is the load vector.

Considering the influence of these two outliers, the global weight $w_{i}=w_{i}^{x} w_{i}^{r}$ of the $i$ th object is recalculated in each iteration step. The new weighted observation value $\left(\sqrt{w_{i}} x_{i}, \sqrt{w_{i}} y_{i}\right)$ is obtained. Then, the BFGS Algorithm 2 can be run on the reweighted model.

The ABFGS algorithm proposed in this paper has the following advantages over the BFGS algorithm in process prediction:

(1) For the data center, the ABFGS algorithm using weighted center is more robust to outliers than the BFGS algorithm using the mean center.

(2) ABFGS accelerates the convergence speed of the outlier weight coefficient. The weight of ABFGS outliers is much smaller than the BFGS algorithm, which ensures the accuracy of the prediction of process data containing outliers. 
3.3. Fault Monitoring Based on ABFGS Algorithm. According to the relationship between input and output, the input is decomposed orthogonally $[12,13]$. Specific methods are as follows:

(1) Perform singular value decomposition (SVD) on matrix $\mathbf{M M}^{T}$ to establish projection matrix $\mathbf{M M}^{T}=\left[\begin{array}{ll}\mathbf{P}_{\mathbf{M}} & \widetilde{\mathbf{P}}_{\mathbf{M}}\end{array}\right]\left[\begin{array}{cc}\Lambda_{\mathbf{M}} & 0 \\ 0 & 0\end{array}\right]\left[\begin{array}{c}\mathbf{P}_{\mathbf{M}}^{T} \\ \widetilde{\mathbf{P}}_{\mathbf{M}}^{T}\end{array}\right], \quad \Pi_{\mathbf{M}}=\mathbf{P}_{\mathbf{M}} \mathbf{P}_{\mathbf{M}}^{T}$, $\Pi_{\mathbf{M}}^{\perp}=\widetilde{\mathbf{P}}_{\mathbf{M}} \widetilde{\mathbf{P}}_{\mathbf{M}}^{T}, \quad \Lambda_{\mathbf{M}} \in \mathbf{R}^{l \times l} . \quad$ Here, $\quad \mathbf{P}_{\mathbf{M}} \in \mathbf{R}^{m \times l}$, $\widetilde{\mathbf{P}}_{\mathbf{M}} \in \mathbf{R}^{m \times(m-l)}$, and $\Pi_{\mathbf{M}}$ and $\Pi_{\mathbf{M}}^{\perp}$ are projection matrices of $\mathbf{X}$.

(2) Decompose the input $\mathbf{X}$ into two orthogonal subspaces $\widehat{\mathbf{X}}=\mathbf{X} \Pi_{\widetilde{A}}=\mathbf{T}_{\widehat{\mathbf{u}}} \mathbf{P}_{\mathbf{M}}^{T}, \mathbf{T}_{\widehat{\mathbf{u}}}=\mathbf{X P}_{\mathbf{M}} \in \mathbf{R}^{N \times l}$, and $\widetilde{\mathbf{X}}=\mathbf{X} \Pi_{\mathbf{M}}^{\perp}=\mathbf{T}_{\widetilde{\mathbf{u}}} \widetilde{\mathbf{P}}_{\mathbf{M}}, \mathbf{T}_{\widetilde{\mathbf{u}}}=\mathbf{X} \widetilde{\mathbf{P}}_{\mathbf{M}} \in \mathbf{R}^{N \times(m-l)}$. The two subspaces represent output-related and output-unrelated monitoring results.

(3) For the new sample point $\mathbf{x} \in \mathbf{R}^{m}$, it can be decomposed into $\widehat{\mathbf{x}} \in \mathbf{R}^{m}$ and $\widetilde{\mathbf{x}} \in \mathbf{R}^{m}$. Then, calculate $\mathbf{t}_{\widehat{\mathbf{x}}}=\mathbf{x} \mathbf{P}_{\mathbf{M}} \in \mathbf{R}^{N \times l}$ and $\mathbf{t}_{\tilde{\mathbf{x}}}=\mathbf{x} \widetilde{\mathbf{P}}_{\mathbf{M}} \in \mathbf{R}^{N \times(m-l)}$.

(4) Calculate the output-related and output-unrelated observation statistics $T_{\widehat{x}}^{2}=\mathbf{t}_{\widehat{x}}^{T}\left(\mathbf{T}_{\widehat{\mathbf{x}}}^{T} \mathbf{T}_{\widehat{\mathbf{x}}} / \mathbf{n}-\mathbf{1}\right) \mathbf{t}_{\widehat{\mathbf{x}}}$ and

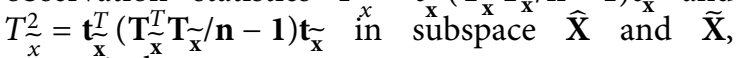
respectively.$$
\mathrm{FAR}=\frac{\text { no. of false alarms }}{\text { total normal samples }} \times 100 \%,
$$$$
\mathrm{FDR}=\frac{\text { no. of effective alarms }}{\text { total faulty samples }} \times 100 \% \text {, }
$$$$
\mathrm{SDR}=\frac{\text { no. of effective alarms in normal data }}{\text { total samples }}+\frac{\text { no. of false alarms in the fault data }}{\text { total samples }} \times 100 \% \text {. }
$$

(5) Calculate the thresholds, where $J_{t h, T_{\curlywedge}^{2}}=\left(\left(N^{2}-1\right) / N(N-l)\right) F_{\alpha}(l, N-l) \quad$ and $J_{t h, T_{\sim}^{2}}^{\stackrel{u}{u}}=\left(\left(N^{2}-1\right) / N(N-l)\right) F_{\alpha}(m-l, N-m-l)$ are the thresholds of output-related statistics $T_{\widehat{x}}^{2}$ and output-unrelated statistics $T_{\widetilde{x}}^{2}$, respectively.

Finally, fault monitoring can be expressed as follows:

(1) When $T_{\hat{x}}^{2}>J_{t h, T_{\hat{x}}^{2}}$, an output-related fault is detected

(2) When $T_{x}^{2}>J_{t h, T_{x}^{2}}$, an output-unrelated fault is detected

Compared with the BFGS algorithm, this algorithm decomposes the input data variables into output-related parts and output-unrelated parts. On this basis, it can construct more favorable statistical information, thus effectively revealing whether the fault is related to the output. Therefore, based on robustness to outliers, the ABFGS algorithm can provide more accurate results for process fault monitoring.

To illustrate the effectiveness of the algorithm, this paper evaluates the performance of the algorithm with the following three evaluation indexes [12].

\section{Case Study of Tennessee Eastman (TE) Process}

4.1. TE Process. TE process has a complex engineering background, involving a large number of random, fuzzy, uncertain, and uncontrollable factors. TE benchmark process is a simulation system based on the actual chemical production process, which provides a practical platform for the evaluation of process control and monitoring methods [26-28]. TE process model consists of five primary units. There are 41 measured values XMEAS (1-41), 12 manipulated variable XMV (1-12), and 8 components: A, B, C, D, E, $\mathrm{F}, \mathrm{G}$, and $\mathrm{h}$, where $\mathrm{g}$ and $\mathrm{h}$ are two by-products.

TE process includes 22 sets of training data and corresponding test data, which are represented by IDV $(i)(i=$ $1,2, \ldots, 22)$ [29]. Except for IDV (0) obtained under normal operating conditions, other 21 sets of data were collected and operated for 48 hours under 21 different fault conditions. 960 sample points were taken from each test set. The fault in the fault set starts from the 161st sample point and ends at the 960th observed sample point. In this paper, 22 process measurements XMEAS (1-22) and 11 manipulated variables
XMV (1-11) are taken as input data matrices [30]. XMEAS (35) represents the product quality of component $g$ and represents the process output. With IDV (0) as the training set, the performance of the ABFGS algorithm is verified by 21 other fault test sets.

4.2. Selection of $m$ Value of Weighted Center. It can be seen from the six figures in Figure 1 that the distance between the weighted center and the real center fluctuates slightly when the value of $m$ is small. But with the increase of the value of $m$, the distance between the two centers tends to be stable. In both cases (a) and (b), the distance between the two centers is the smallest when $m=2$. In four cases (c), (d), (e), and (f), the distance between the two centers is the smallest when $m=1$. Therefore, the ratio of outliers and whether outliers are larger or smaller than normal values affect the value of $m$.

4.3. Simulation of Output Correlation Prediction Performance. The ABFGS algorithm uses the weighted center to determine the center of data, while the standard BFGS algorithm uses the mean center in the same process. In order to show that 
(1) Give the initial point $\mathbf{B}_{0} \in \mathbf{R}^{m}$, the initial quasi-Newton matrix $\mathbf{H}_{0} \in \mathbf{R}^{m \times m}$, and the termination limit $\varepsilon>0$, iterations $k=0$.

(2) If $\left\|\mathbf{g}_{k}\right\| \leq \varepsilon$, stop.

(3) Calculate $\mathbf{d}_{k}=-\mathbf{H}_{k} \mathbf{g}_{k}$.

(4) Let $\mathbf{v}_{k}=\mathbf{d}_{k} /\left\|\mathbf{d}_{k}\right\|$, calculate the score vector $\mathbf{t}_{k}=\mathbf{X} \mathbf{v}_{k}$, and load vector $\mathbf{p}_{k}=\mathbf{X}^{T} \mathbf{t}_{k} / \mathbf{t}_{k}^{T} \mathbf{t}_{k}$. Among them, $\mathbf{t}_{k}$ is the $k$ column of $\mathbf{T}$, and $\mathbf{p}_{k}$ is the $k$ column of $\mathbf{P}$. The $k$ th component $\mathbf{b}_{k}=\mathbf{Y}^{T} \mathbf{t}_{k} / \mathbf{t}_{k}^{T} \mathbf{t}_{k}$ of the load vector $b$ is calculated. Calculate load vector.

(5) Find step factor $\alpha_{k}$ by linear search. Make $\mathbf{B}_{k+1}=\mathbf{B}_{k}+\alpha_{k} \mathbf{d}_{k}$.

(6) Correction $\mathbf{H}_{k}$ produces $\mathbf{H}_{k+1}$,

$\mathbf{H}_{k+1}=\left(\mathbf{E}-\left(\mathbf{s}_{k} \mathbf{y}_{k}^{T} / \mathbf{s}_{k}^{T} \mathbf{y}_{k}\right)\right) \mathbf{H}_{k}\left(\mathbf{E}-\left(\mathbf{y}_{k} \mathbf{s}_{k}^{T} / \mathbf{s}_{k}^{T} \mathbf{y}_{k}\right)\right)+\left(\mathbf{s}_{k} \mathbf{s}_{k}^{T} / \mathbf{s}_{k}^{T} \mathbf{y}_{k}\right)$.

$\mathbf{H}_{k+1}$ satisfies the quasi-Newton condition $\mathbf{H}_{k+1} \mathbf{y}_{k}=\mathbf{s}_{k}$, where

$\mathbf{y}_{k}=\mathbf{g}_{k+1}-\mathbf{g}_{k}, \mathbf{s}_{k}=\mathbf{B}_{k+1}-\mathbf{B}_{k}$.

(7) $k=k+1$, go to Step 2 .

AlgORITHM 1: BFGS algorithm.

(1) Calculate the robust starting value of weight $w_{i}=w_{i}^{x} w_{i}^{r}$. The residual weight $w_{i}^{r}$ is obtained by $r_{i}=y_{i}-\bar{e}(\mathbf{y})$ and (12). The leverage weight $w_{i}^{x}$ is calculated by (11), and the score vector is replaced by $x_{i}$.

(2) The $i$ th rows of $\mathbf{X}$ and $\mathbf{y}$ are multiplied by $\sqrt{w_{i}}(i=1,2, \ldots, N)$ to obtain weighted data matrices $\mathbf{X}_{1}$ and $\mathbf{y}_{1}$. BFGS regression analysis is performed on the new weighted data matrices $\mathbf{X}_{1}$ and $\mathbf{y}_{1}$. The updated score matrix $\mathbf{T}$ is obtained from the regression analysis results. Then, each row of the new matrix $\mathbf{T}$ is corrected by dividing $\sqrt{w_{i}}$. The $\mathbf{b}$ vector of the original problem is calculated by the modified score matrix $\mathbf{T}$.

(3) Update residual $r_{i}$ by formula $r_{i}=y_{i}-\mathbf{t}^{i} \mathbf{b}$. Then, use $r_{i}$ to correct the weight of $w_{i}$.

(4) Repeat steps 2 and 3 until $\mathbf{b}$ converges. As long as the norm of the difference between two successive approximations of $\mathbf{b}$ is less than the specified threshold (e.g., 0.01), the iteration will stop. Go to Step 5.

(5) The final regression coefficient vector $\mathbf{M}=\mathbf{P b}$ is obtained through the last ABFGS regression step. The final prediction function is $y_{i}=\mathbf{x}_{i} \mathbf{M}$. Here, $\mathbf{x}_{i}$ is the new sample point and $y_{i}$ is the corresponding output prediction. Establish an online output prediction scheme by using the prediction function.

Algorithm 2: ABFGS algorithm.

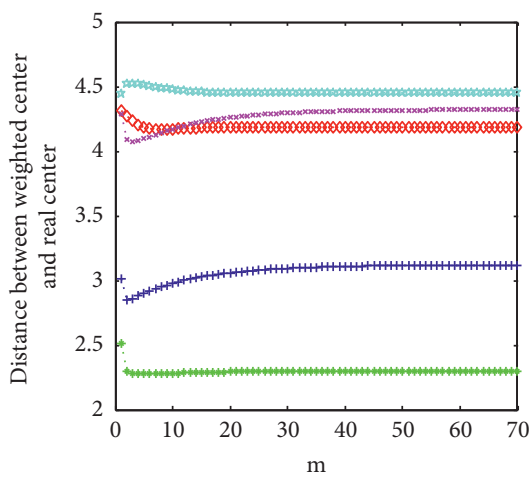

(a)

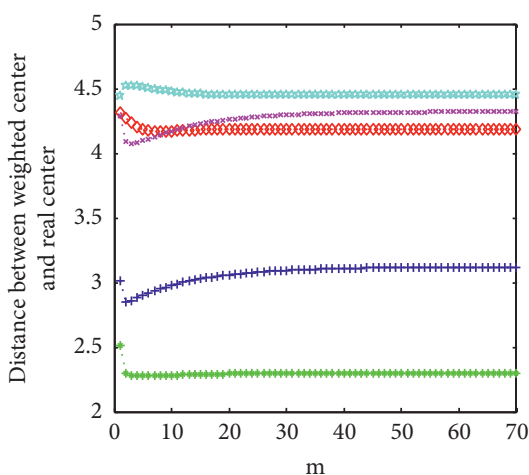

(b)

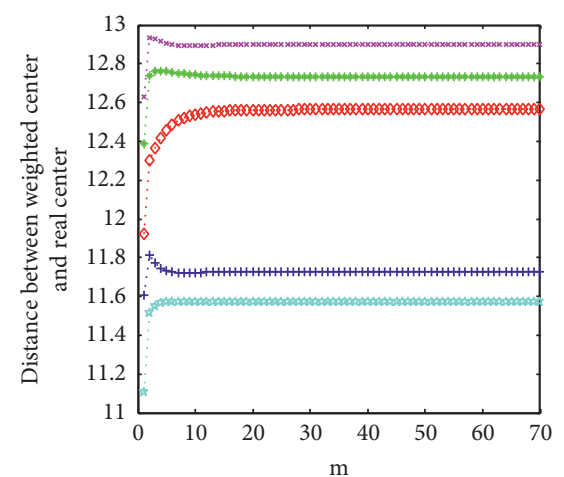

(c)

Figure 1: Continued. 


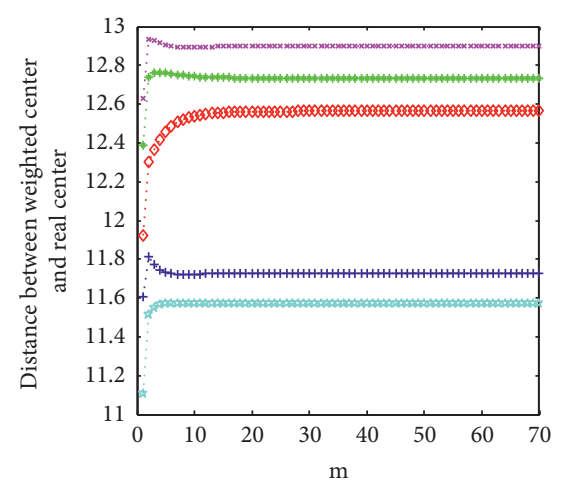

(d)

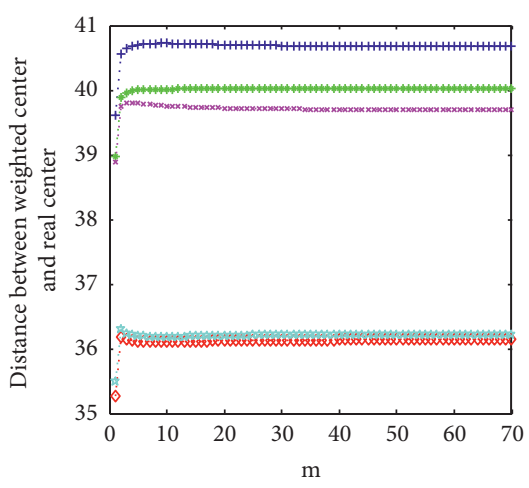

(e)

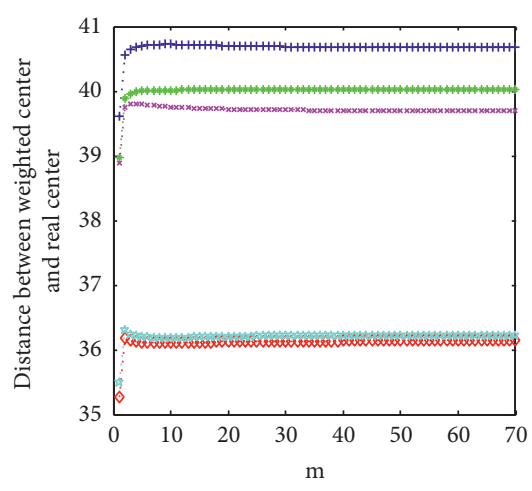

(f)

FIGURE 1: Influence of different values of the parameter $m$ in the weighted center on the distance between the weighted center and real center. Each graph contains five curves with random outliers. (a) The normal point contains a $5 \%$ abnormal value, and the abnormal value is 0.5 of the normal value. (b) The normal point contains a $10 \%$ abnormal value, and the abnormal value is 0.5 of the normal value. (c) The normal point contains a $15 \%$ abnormal value, and the abnormal value is 0.5 of the normal value. (d) The normal point contains a $5 \%$ abnormal value, and the abnormal value is 1.5 of the normal value. (e) The normal point contains a $10 \%$ abnormal value, and the abnormal value is 1.5 of the normal value. (f) The normal point contains a $15 \%$ abnormal value, and the abnormal value is 1.5 of the normal value.

the ABFGS algorithm (with the weighted center) is more robust to outliers than the BFGS algorithm (with the mean center), we introduce a certain percentage of outliers to available measured values in simulation. Because of the physical limitations of sensors, outliers can be higher or lower than normal values [19]. Therefore, we set the outliers to 0.5 times and 1.5 times of normal data. The ratio of outliers is $5 \%, 10 \%$, and $15 \%$ respectively.

Take the first two columns of the input data to form a two-dimensional variable space. Figures 2(a)-2(f), respectively, show various centers of data: the weighted center proposed in this paper, total square loss center (tSL-center) [19], mean center with anomalies, and true center without outliers. It can be seen from the figure that the data center (weighted center) estimated by ABFGS is closer to the real center than other centers. The weighted center is not affected by whether the outliers are enlarged or reduced. The tSLcenter is closer to the real center than the mean center when the outliers are enlarged. But if the outliers are reduced, the tSL-center will be farther away from the real center than the mean center. That is, the tSL-center will be affected by whether the outliers are enlarged or reduced. In other words, the weighted center of data based on ABFGS is far away from outliers, while the rest centers are closer to outliers (such as the mean center used by BFGS). This shows that ABFGS is more robust to outliers than BFGS. With the increase of the ratio of outliers, this advantage of ABFGS becomes more obvious.

We use the training data collected from normal operating conditions to consider the prediction performance of the ABFGS algorithm. Specifically, the training set is selected as half of IDV (0) samples. In order to analyze the robustness of ABFGS to prediction performance, $5 \%, 10 \%$, and $15 \%$ outliers were added to normal data (enlarged to 1.5 times of normal value). Select the other half of IDV (0) as the predicted test data. The simulation results are shown in Figures 3(a), 3(b), and 3(c). The black, red, and blue lines, respectively, represent the actual measured value of XMEAS
(35), the predicted value of the ABFGS algorithm, and the BFGS algorithm. It can be seen that, in all cases, the prediction based on ABFGS is closer to the actual measured value. However, the prediction based on BFGS gradually deviates from the actual measured value with the increase of the ratio of outliers. Therefore, the ABFGS algorithm has better prediction performance than the BFGS algorithm and shows stronger robustness when the ratio of outliers increases.

At last, when $5 \%, 10 \%, 15 \%, 20 \%, 25 \%, 30 \%, 35 \%$, and $40 \%$ of the data are taken as outliers, we simulated the mean squared error (MSE) between the predicted value and the real measured value of BFGS algorithm and ABFGS algorithm, respectively. The simulation results are shown in Figure 4. It can be seen from Figure 4 that the MSE of BFGS increases with the increase of abnormal value percentage. In contrast, the minimum mean square error (MSE) of ABFGS is quite small and almost zero. In all cases, the MSE value of BFGS is always higher than that of ABFGS. The difference between them gradually increases with the increase of the outlier ratio. This is because when outliers appear, ABFGS can reduce the weight of outliers by robust data scaling and weaken the influence of outliers on the algorithm. However, for BFGS, it has no way to deal with potential outliers but let them affect the construction of the model, so it is not robust to outliers.

It can be seen from the above results that when there are outliers in the predictor space, compared with BFGS, ABFGS has better prediction ability and stronger robustness to outliers.

4.4. Simulation of Output-Related Monitoring Performance. In this part, we will use the ABFGS algorithm, BFGS algorithm, kernel independent component analysis (KICA) algorithm [31-34], and PLS algorithm [35-37] to monitor 21 kinds of faults in the TE process and compare their performances. Since faults in measurable variable space may 

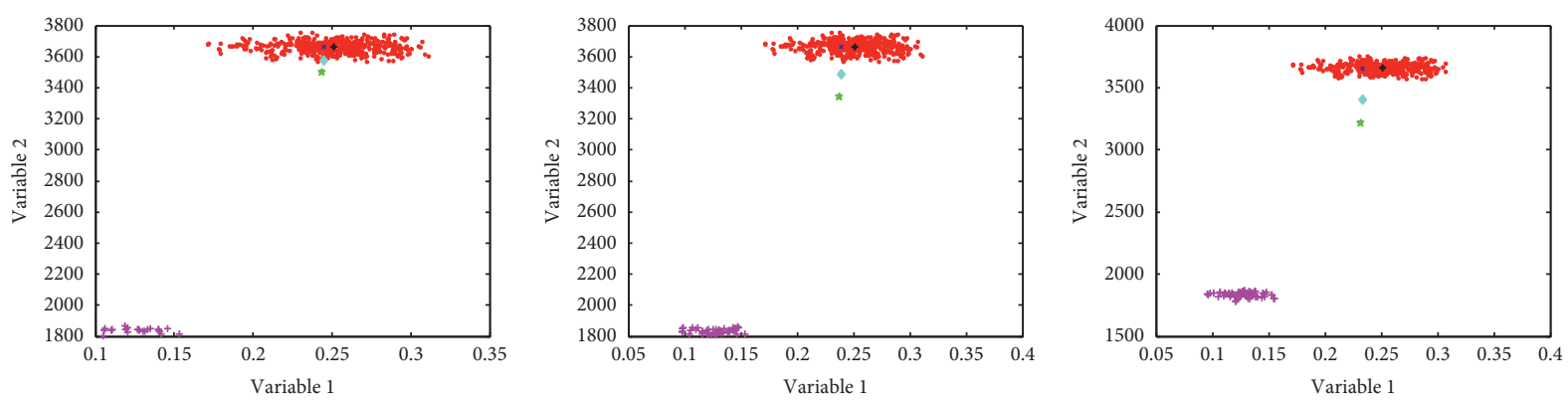

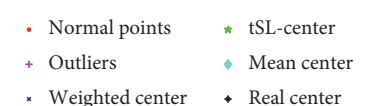

(a)

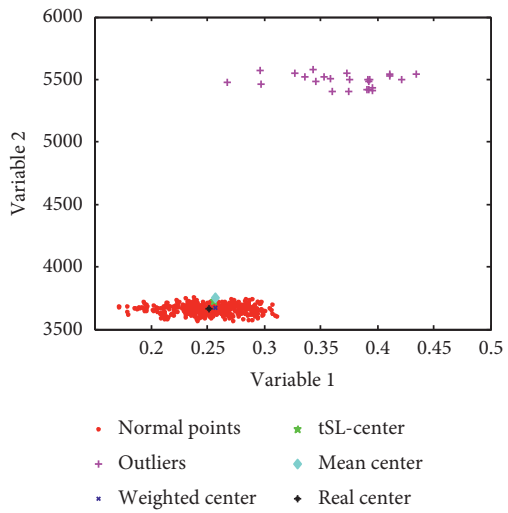

(d)

\begin{abstract}
- Normal points * tSL-center
+ Outliers

Weighted center
\end{abstract}

(b)

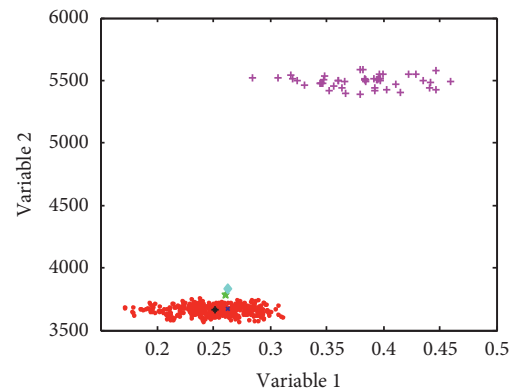

$$
\begin{array}{ll}
\text { - Normal points } & * \text { tSL-center } \\
+ \text { Outliers } & * \text { Mean center } \\
* \text { Weighted center } & \text { - Real center }
\end{array}
$$

(e)

$$
\begin{array}{ll}
\text { - Normal points } & * \text { tSL-center } \\
\text { + Outliers } & \text { - Mean center } \\
* \text { Weighted center } & \text { + Real center }
\end{array}
$$

(c)

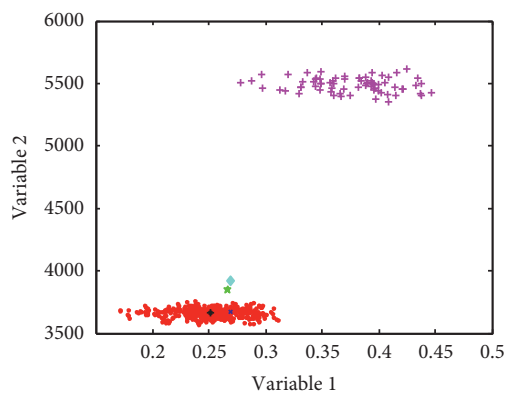

$$
\begin{array}{ll}
\text { - Normal points } & * \text { tSL-center } \\
+ \text { Outliers } & * \text { Mean center } \\
* \text { Weighted center } & \text { * Real center }
\end{array}
$$

(f)

Figure 2: Estimation of data centers of a $2 \mathrm{D}$ variable space. (a) The normal point contains a $5 \%$ abnormal value, and the abnormal value is 0.5 of the normal value. (b) The normal point contains a $10 \%$ abnormal value, and the abnormal value is 0.5 of the normal value. (c) The normal point contains a $15 \%$ abnormal value, and the abnormal value is 0.5 of the normal value. (d) The normal point contains a $5 \%$ abnormal value, and the abnormal value is 1.5 of the normal value. (e) The normal point contains a $10 \%$ abnormal value, and the abnormal value is 1.5 of the normal value. (f) The normal point contains a $15 \%$ abnormal value, and the abnormal value is 1.5 of the normal value.

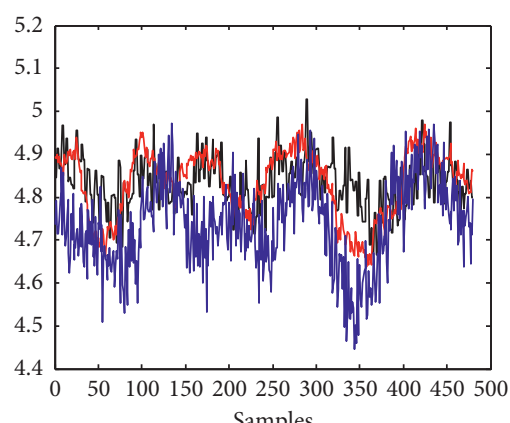

Samples

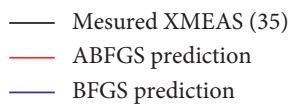

(a)

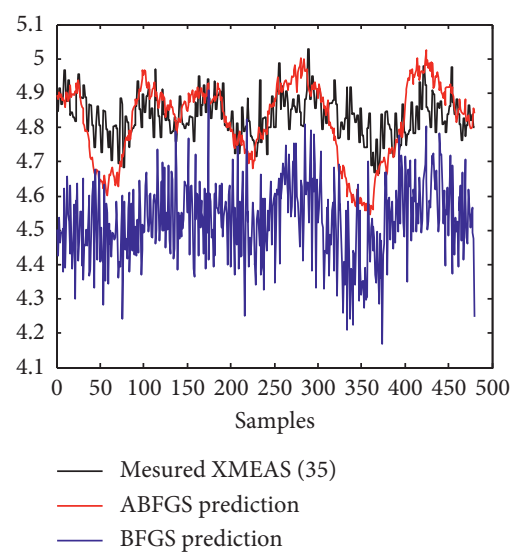

(b)

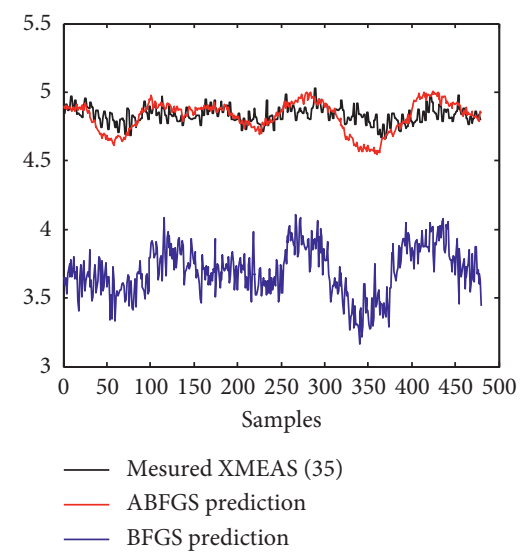

(c)

FIGURe 3: Using ABFGS and BFGS to predict output correlation. (a) The normal data contains 5\% abnormal values. (b) The normal data contains $10 \%$ abnormal values. (c) The normal data contains $15 \%$ abnormal values. 


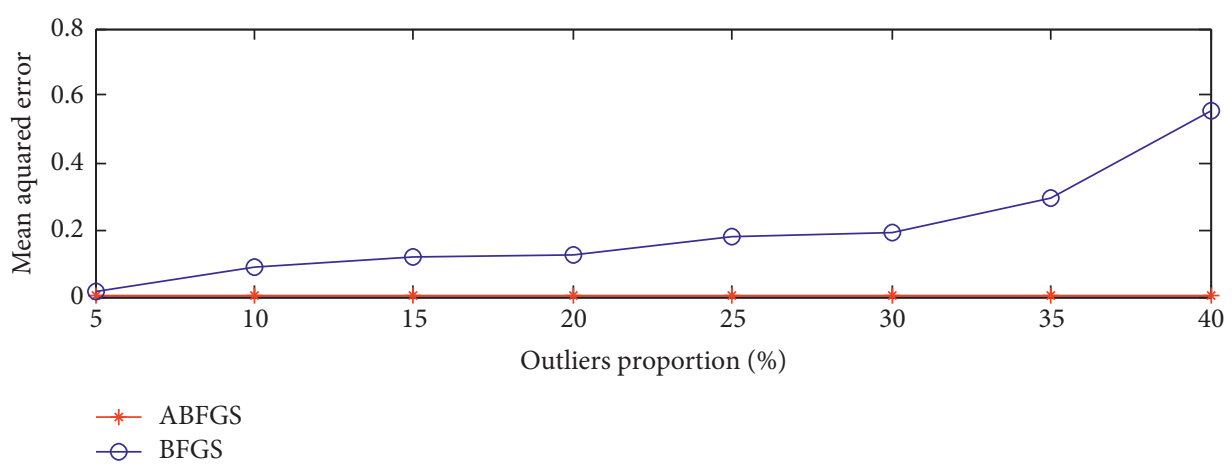

FIGURE 4: The MSE of ABFGS and BFGS with different ratios of outliers are simulated.

occur in different subspaces, it is particularly important to choose the appropriate monitoring scheme according to relevant test statistics. In this part, IDV (0) is used as the training set to build the prediction model, and the other 21 fault test sets are used to verify the monitoring efficiency of the model. In the simulation, we added $25 \%$ outliers to the training set IDV (0), assuming that the outliers are 1.5 times the normal data. According to [13], the number of latent variables of the first three methods is set to 6 . The KICA algorithm has two independent components.

Firstly, according to the control line of whether the output exceeds the PauTa criterion, whether the fault is related to the output is defined. The fault monitoring performance of the BFGS algorithm and ABFGS algorithm is compared through the relationship between fault and output. The simulation results of fault $\operatorname{IDV}(1), \operatorname{IDV}(5)$, and IDV(19) monitored by BFGS algorithm and ABFGS algorithm are shown in Figures 5-7, respectively.

Figure 5(a) shows the influence of fault IDV (1) on the output. The output is affected by the fault from the 161st sample, the fault is self-repairing after the 480th sample, and the output is no longer affected by the fault. Figure 5(b) shows the monitoring results of the main part $\left(T^{2}\right)$ and the residual part (SPE) of the BFGS algorithm. It can be seen that after the fault occurs, the fault continuously appears in the two monitoring results, but there is no way to judge whether IDV (1) has an influence on the output. Figure 5(c) shows the output-related and output-unrelated indexes of the ABFGS algorithm. From the monitoring results, it can be seen that the output-related part has been monitored for faults between 161 sample points and 480 sample points. After the 480th sample point, the output-related parts basically returned to normal, and only a few sample points showed faults. The output-unrelated part shows that the fault always exists from the beginning of 161 sample points to the end of sample points. By comparison, ABFGS has superior performance. It can not only describe that the fault occurred in the output-related and output-unrelated parts but also clearly reveal that the influence of IDV (1) on the output is between the 161st and 480th samples.

Figure 6(a) shows the influence of fault IDV (5) on the output. It can be seen from the image that only some sample points between the 161 st and 480th sample points are affected by the fault IDV (5). Figure 6(b) shows the monitoring results of the main part $\left(T^{2}\right)$ and residual part (SPE) of the BFGS algorithm. It can be seen that both parts can indicate that some sample points between the 161st and 480th have faults, and other sample points are not affected by faults. Figure 6(c) shows the output-related and output-unrelated indexes of the ABFGS algorithm. It can be seen from the monitoring results that there is a fault in the output-related part between the 161st and 480th sampling points, and the output-related part returns to normal after the 480th sampling point. Output-unrelated part shows that faults always exist from the 161st sampling point to the end of the sampling point. The ABFGS algorithm can monitor the whole range of faults and can clearly point out which sampling points have output-related faults and which sampling points have output-unrelated faults.

Figure 7(a) shows the influence of the fault IDV (19) on the output, and the output is not affected by the fault from the beginning to the end, so it is an output irrelevant fault. Figure 7(b) shows the monitoring results of the main part $\left(T^{2}\right)$ and residual part (SPE) of the BFGS algorithm. Both monitoring results indicate that only a few sample points have a fault. Figure 7 (c) shows the output-related and output-unrelated indexes of the ABFGS algorithm. From the monitoring results, it can be seen that only a few sample points in the output-related part have detected faults. Output irrelevant part shows that almost all sample points are in fault state from the beginning of 161 sample points to the end of sample points. That is to say, ABFGS can not only monitor the faults that BFGS cannot monitor but also indicate that the faults occur in the output-unrelated part.

Table 1 lists the monitoring effects of four methods (the training data contains $25 \%$ outliers), including the KICA algorithm with the best fault monitoring effect at present. The first 21 rows of the table reflect the failure detection rate, and the 22 nd row reflects the average failure detection rate. It can be seen from the columns corresponding to the PLS algorithm and BFGS algorithm that although both methods belong to the conjugate direction method, the BFGS algorithm is the quasi-Newton method, which has a higher fault detection rate than the PLS algorithm. The first block in the table is the fault related to the output. Except for IDV (2), the highest fault monitoring rate is given by the ABFGS algorithm proposed in this paper. The fault monitoring rate of IDV (2) given by the ABFGS algorithm is 0.5 percentage 


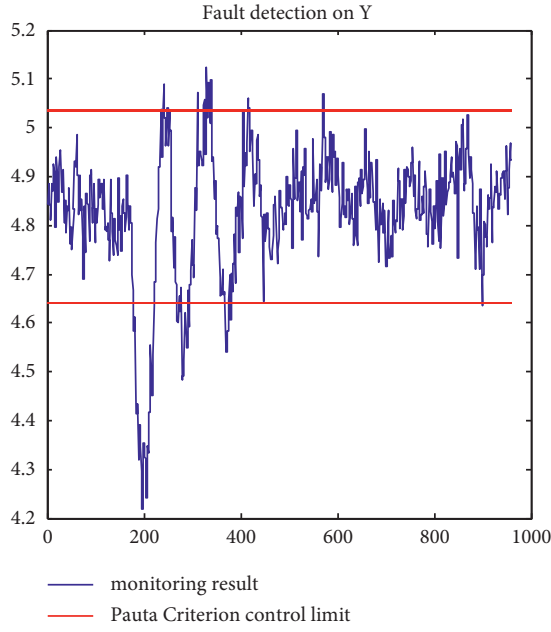

(a)
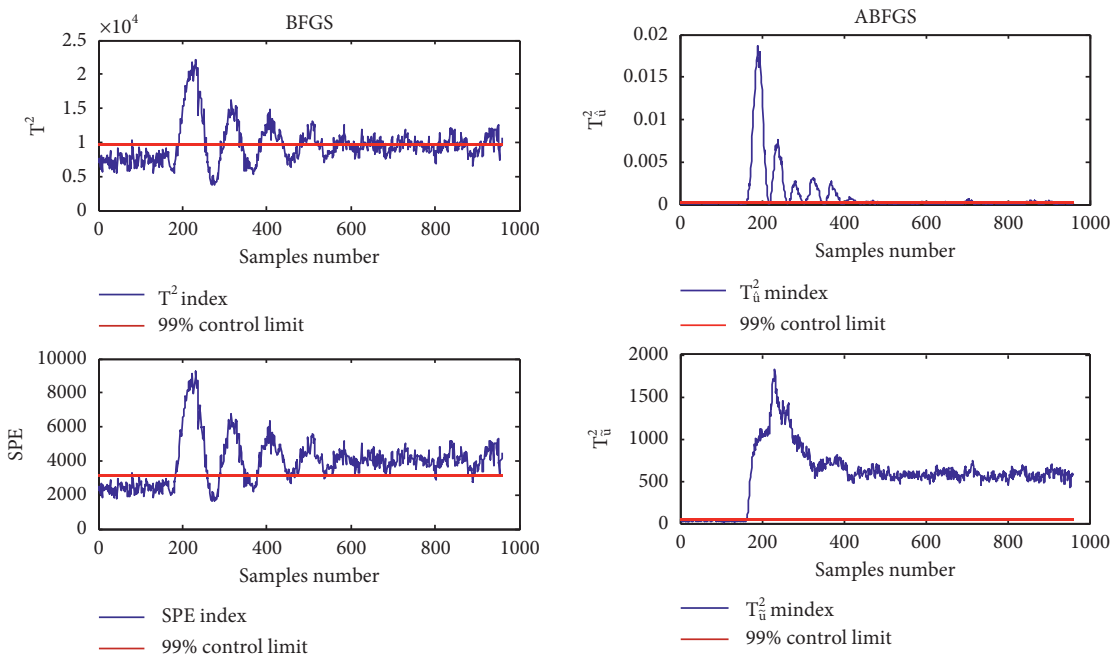

(b)

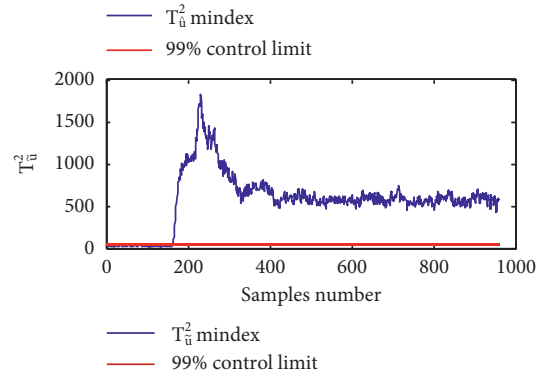

(c)

FIGURE 5: Using the BFGS algorithm and ABFGS algorithm to monitor the fault IDV of TE process (3). (a) Output fault monitoring. (b) BFGS algorithm monitoring results. (c) ABFGS algorithm monitoring results.

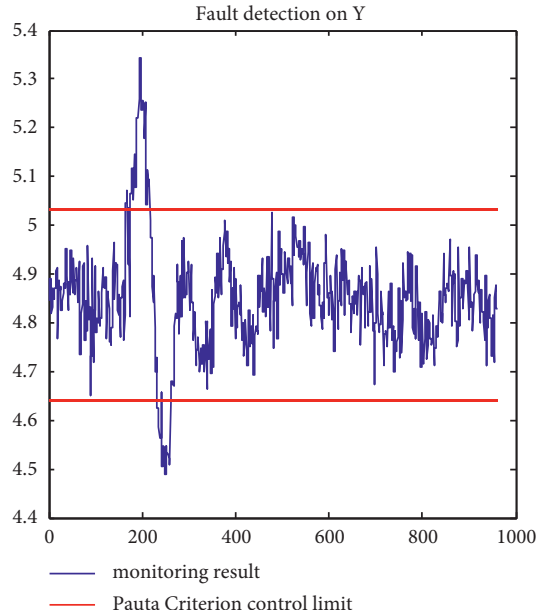

(a)
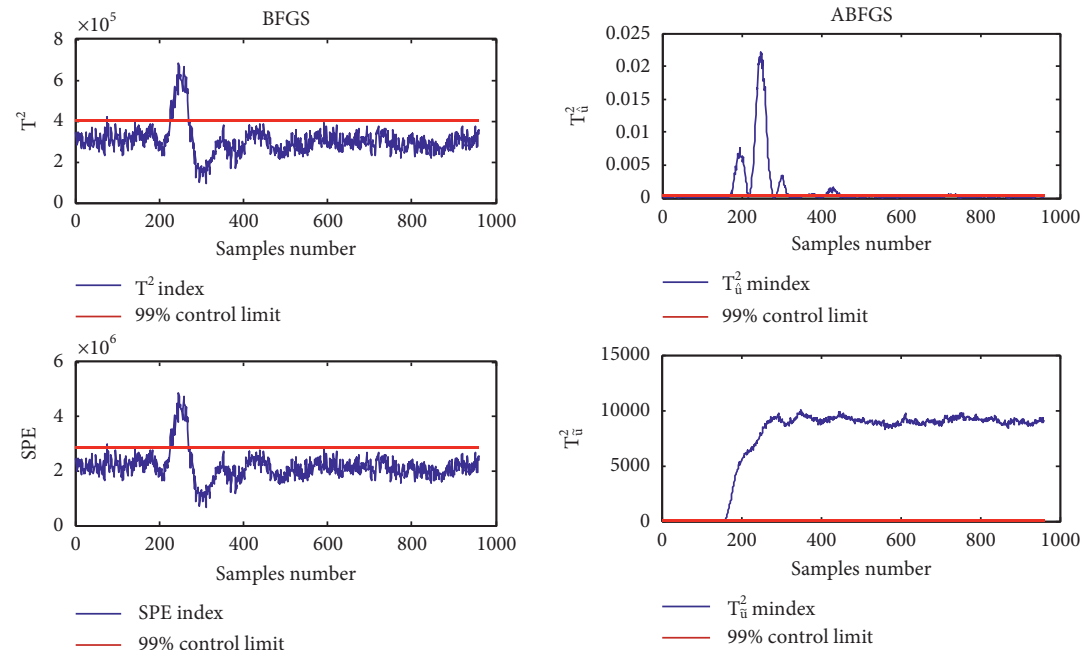

(b)

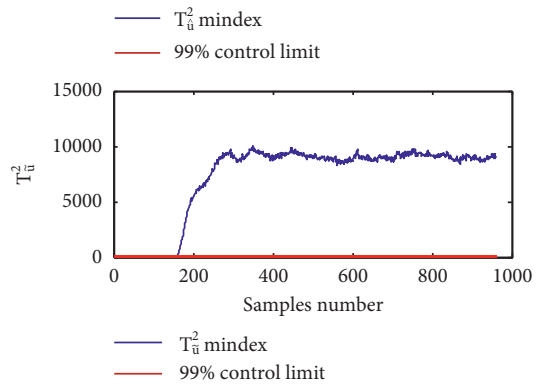

(c)

FIGURE 6: Using BFGS algorithm and ABFGS algorithm to monitor the fault IDV (5) of TE process (8). (a) Output fault monitoring. (b) BFGS algorithm monitoring results. (c) ABFGS algorithm monitoring results.

points less than the highest fault monitoring rate. The second block in the table is the output-unrelated fault. Except for IDV (3) and IDV (15), the ABFGS algorithm gives the highest fault monitoring rate. The average fault monitoring rate of ABFGS is also the highest. The false alarm rate is shown in the last row of the table; the false alarm rate of the ABFGS algorithm is not the lowest but lower than that of the KICA algorithm with the second highest fault monitoring rate. The comparison between the four methods shows that the ABFGS method has the strongest robustness.

Figure 8 shows the successful detection rate (SDR) of 21 faults by four methods. The ABFGS algorithm has an SDR of over $90 \%$ for 15 faults and an SDR of over $80 \%$ for 16 faults. Except for fault IDV (3) and IDV (15), the ABFGS algorithm has the highest SDR. SDR of fault IDV (3) ABFGS algorithm is slightly lower than other methods. The fault IDV (15) ABFGS algorithm and KICA algorithm basically have the same SDR value, which is higher than that of the other two methods. At the same time, the SDR values of fault IDV (3), IDV (9), and IDV (15) are all very low, which shows that they cannot effectively detect such faults.

Based on the above simulation results and discussions, it is found that the fault monitoring based on the ABFGS algorithm can not only reveal the time and possibility of the fault but also distinguish whether the fault affects the output index. A timely and accurate fault alarm prompt operator system failure, can largely avoid the waste of raw materials, and reduce the production of defective products. Therefore, the ABFGS algorithm 


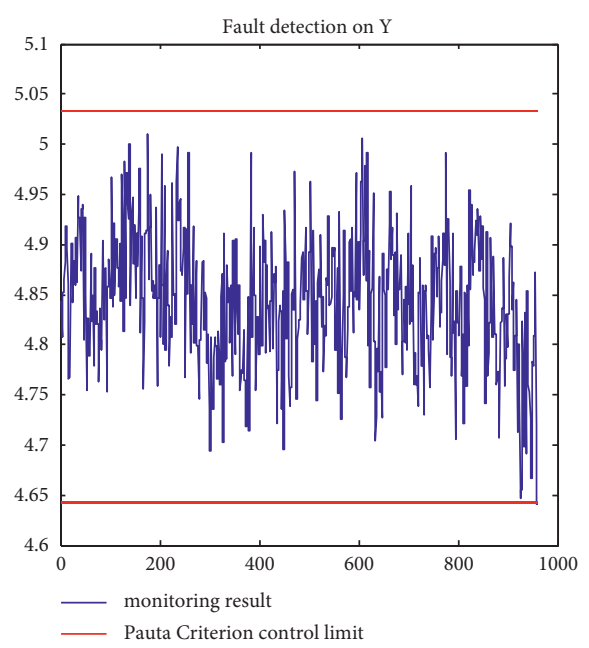

(a)
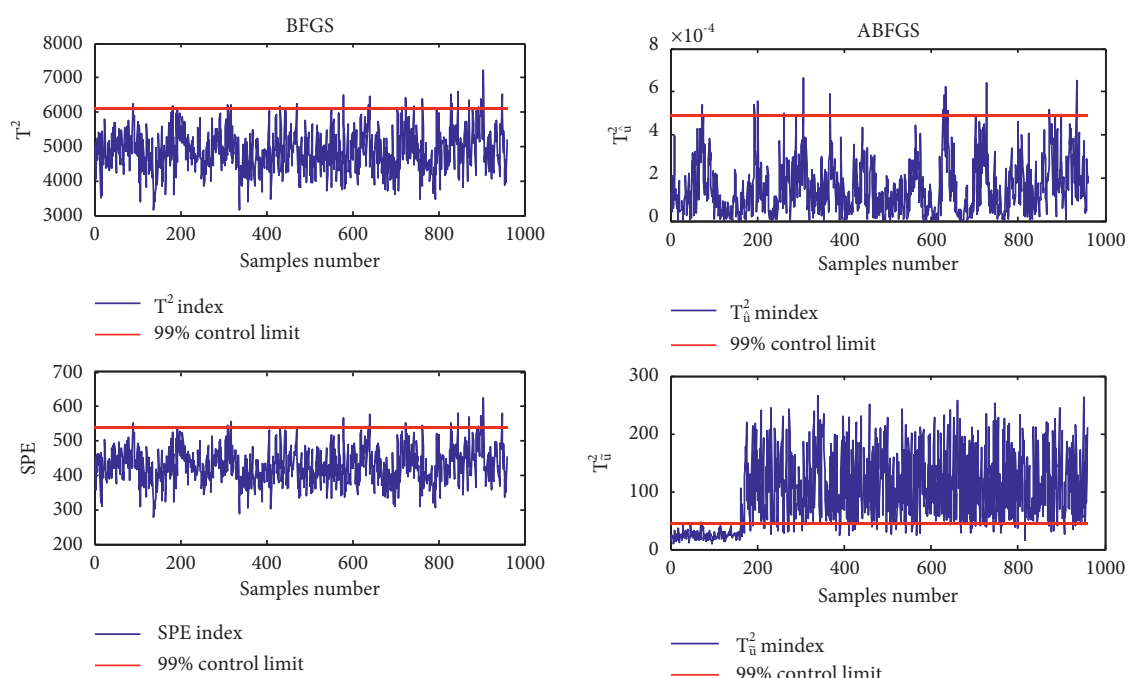

(b) (c)

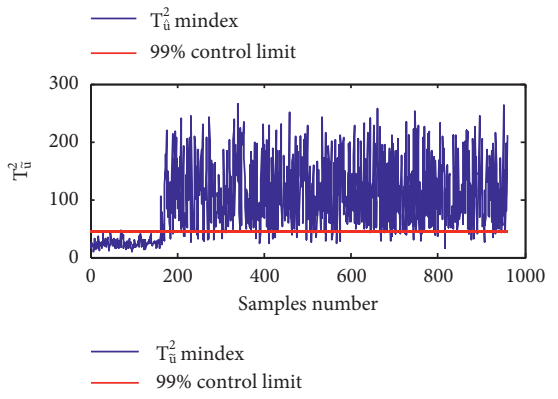

FIGURE 7: Using BFGS algorithm and ABFGS algorithm to monitor the fault IDV (19) of TE process (8). (a) Output fault monitoring. (b) BFGS algorithm monitoring results. (c) ABFGS algorithm monitoring results.

TABLE 1: FDRs of the 21 faults in the TE benchmark (\%).

\begin{tabular}{|c|c|c|c|c|c|c|c|c|}
\hline \multirow{2}{*}{ Fault } & \multicolumn{2}{|c|}{ PLS } & \multicolumn{2}{|c|}{ BFGS } & \multicolumn{2}{|c|}{ KICA } & \multicolumn{2}{|c|}{ ABFGS } \\
\hline & $T^{2}$ & SPE & $T^{2}$ & SPE & $T^{2}$ & SPE & $T_{\widehat{U}}^{2}$ & $T_{\widetilde{U}}^{2}$ \\
\hline 1 & 0.00 & 0.00 & 50.75 & 88.63 & 98.75 & 98.75 & 17.88 & 99.88 \\
\hline 2 & 0.00 & 0.00 & 94.00 & 92.25 & 98.63 & 98.50 & 96.75 & 98.13 \\
\hline 5 & 5.50 & 5.38 & 6.25 & 6.13 & 20.50 & 21.38 & 8.25 & 100.00 \\
\hline 6 & 30.25 & 28.88 & 79.00 & 79.50 & 97.25 & 98.25 & 95.63 & 100.00 \\
\hline 7 & 6.75 & 6.88 & 9.00 & 9.38 & 37.88 & 25.00 & 18.13 & 100.00 \\
\hline 8 & 32.50 & 32.13 & 38.75 & 40.13 & 73.63 & 87.50 & 39.38 & 98.13 \\
\hline 10 & 6.88 & 6.63 & 10.75 & 10.63 & 30.88 & 9.50 & 2.25 & 84.63 \\
\hline 12 & 21.38 & 20.25 & 22.38 & 22.50 & 68.38 & 61.75 & 58.00 & 99.88 \\
\hline 13 & 41.38 & 41.00 & 44.00 & 43.63 & 88.63 & 81.50 & 61.25 & 95.25 \\
\hline 16 & 0.25 & 0.25 & 1.13 & 1.00 & 5.38 & 1.75 & 1.25 & 89.25 \\
\hline 17 & 5.00 & 5.00 & 9.50 & 10.00 & 7.38 & 0.75 & 62.75 & 96.88 \\
\hline 18 & 30.75 & 28.88 & 75.00 & 75.25 & 82.38 & 86.00 & 84.25 & 89.88 \\
\hline 20 & 15.13 & 14.88 & 16.75 & 18.75 & 10.38 & 5.00 & 6.75 & 89.88 \\
\hline 21 & 10.63 & 10.88 & 29.38 & 27.75 & 36.75 & 18.50 & 0.38 & 43.38 \\
\hline 3 & 4.13 & 4.13 & 4.00 & 4.00 & 10.63 & 2.38 & 0.13 & 0.38 \\
\hline 4 & 0.00 & 0.00 & 1.50 & 1.50 & 4.25 & 0.63 & 22.00 & 100.00 \\
\hline 9 & 0.50 & 0.50 & 0.38 & 0.25 & 0.63 & 0.25 & 1.38 & 2.63 \\
\hline 11 & 1.25 & 1.25 & 2.25 & 2.38 & 14.25 & 0.38 & 10.88 & 71.38 \\
\hline 14 & 8.13 & 8.25 & 9.75 & 10.13 & 0.88 & 2.38 & 38.63 & 100.00 \\
\hline 15 & 1.63 & 1.63 & 2.13 & 2.00 & 6.13 & 0.88 & 4.50 & 2.00 \\
\hline 19 & 1.00 & 1.00 & 2.38 & 2.13 & 2.25 & 1.75 & 2.25 & 90.13 \\
\hline AVG & 10.62 & 10.37 & 24.24 & 26.09 & 37.89 & 33.46 & 30.13 & 78.65 \\
\hline AVG-FAR & 0.63 & 0.63 & 0.54 & 0.57 & 0.95 & 1.04 & 0.86 & 0.74 \\
\hline
\end{tabular}

The bold data in the table represent the highest fault detection rate. 


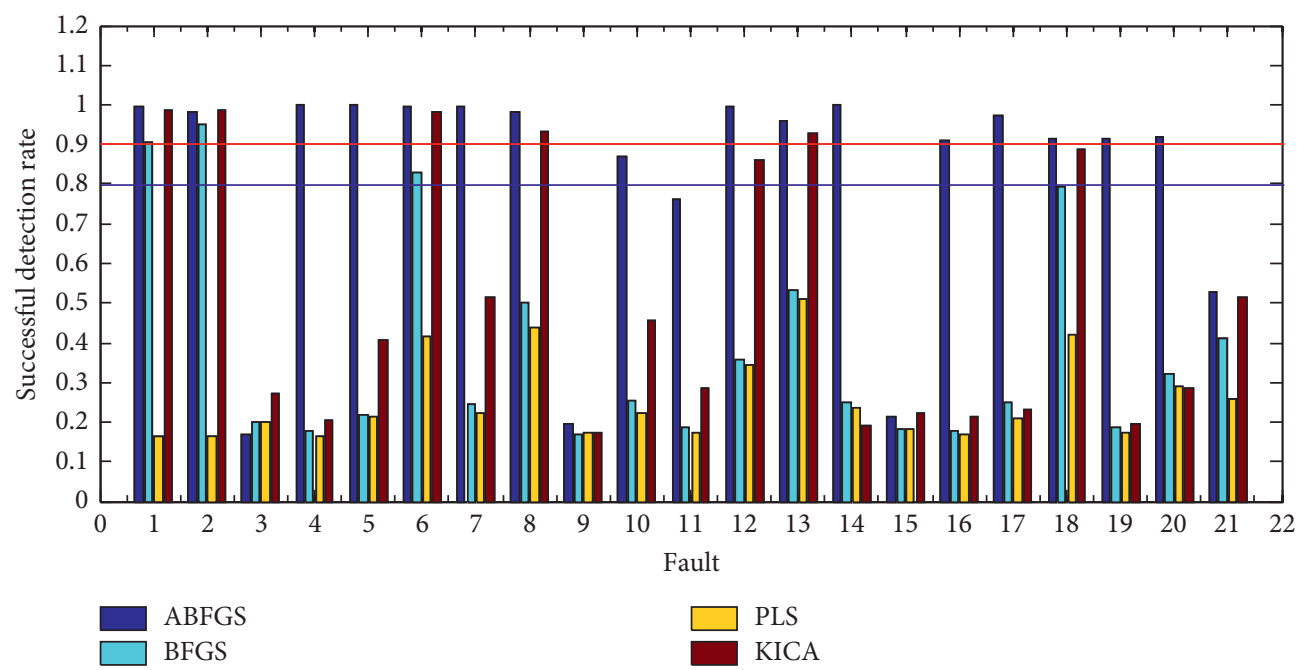

FIGURE 8: SDR of 21 faults in TE process.

has an excellent ability in monitoring process faults. Combined with the analysis of the ABFGS algorithm in process prediction, it can be concluded that the proposed ABFGS algorithm has good monitoring performance for the TE benchmark process in prediction and output-related fault monitoring.

\section{Conclusion}

In this paper, a robust ABFGS algorithm is proposed, which is used for output prediction and output-related fault monitoring. Data centralization is a key step that affects the performance of the algorithm. The central formula given in this paper is basically unaffected by outliers, which ensures the robustness of the ABFGS algorithm in this paper. Therefore, the ABFGS algorithm can predict the output more accurately. In addition, in the aspect of fault monitoring, the BFGS algorithm can only monitor faults, while the ABFGS algorithm proposed in this paper can not only monitor faults but also indicate whether faults are related to output. Accurate prediction and timely fault monitoring can avoid casualties and significant economic losses. Finally, the simulation results of the TE benchmark process show that the ABFGS algorithm does have the above advantages. Compared with the other three algorithms, the ABFGS algorithm can not only give out whether the fault is related to the output but also provide a higher fault monitoring rate, which can provide a better reference for industrial systems.

The deficiency of this method is that the BFGS method has a certain dependence on the initial point, and it needs to train a better initial point. In addition, this method can only monitor the occurrence of the fault but does not specifically locate the fault, which is also a research direction in the future.

\section{Data Availability}

The data in this article are obtained from the following website: https://web.mit.edu/braatzgroup/links.html.

\section{Conflicts of Interest}

The authors declare that there are no conflicts of interest.

\section{Acknowledgments}

This work was supported by the State Key Laboratory of Synthetically Automation for Process Industries Fundamental Research Funds of China (No. 2013ZCX02), the National Natural Science Foundation of China (No. 61903068), and the National Key Research and Development Program of China (No. 2020AAA0109203).

\section{References}

[1] X. Jin, J. Fan, and T. W. S. Chow, "Fault detection for rollingelement bearings using multivariate statistical process control methods," IEEE Transactions on Instrumentation and Measurement, vol. 68, no. 9, pp. 3128-3136, 2019.

[2] G. Yang and X. Gu, "Fault diagnosis of complex chemical processes based on enhanced naive Bayesian method," IEEE Transactions on Instrumentation and Measurement, vol. 69, no. 7, pp. 4649-4658, 2020.

[3] M. G. Xibilia, M. Latino, Z. Marinkovic, A. Atanaskovic, and N. Donato, "Soft sensors based on deep neural networks for applications in security and safety," IEEE Transactions on Instrumentation and Measurement, vol. 69, no. 10, pp. 7869-7876, 2020.

[4] S. X. Ding, Model-Based Fault Diagnosis Techniques, Springer, Berlin, Germany, 2008.

[5] M. F. Harkat, M. Mansouri, M. Nounou, and H. Nounou, "Fault detection of uncertain nonlinear process using intervalvalued data-driven approach," Chemical Engineering Science, vol. 205, no. 21, pp. 36-45, 2019.

[6] W. Yan, G. Li, Z. Wu, S. Wang, and P. S. Yu, "Extracting diverse-shapelets for early classification on time series," World Wide Web, vol. 23, no. 6, pp. 3055-3081, 2020.

[7] G. Wang and S. Yin, "Quality-related fault detection approach based on orthogonal signal correction and modified PLS," IEEE Transactions on Industrial Informatics, vol. 11, no. 2, pp. 398-405, 2015. 
[8] T. Lan, C. D. Tong, X. X. Chen, X. H. Shi, and Y. W. Chen, "KPI relevant and irrelevant fault monitoring with neighborhood component analysis and two-level PLS," Journal of the Franklin Institute, vol. 355, no. 16, pp. 8049-8064, 2018.

[9] K. Zhang, Y. A. W. Shardt, Z. Chen, X. Yang, S. X. Ding, and K. Peng, "A KPI-based process monitoring and fault detection framework for large-scale processes," ISA Transactions, vol. 68, pp. 276-286, 2017.

[10] R. R. Sun, "Multiblock global orthogonal projections to latent structures for fault diagnosis," Chemometrics and Intelligent Laboratory Systems, vol. 204, no. 15, pp. 1-17, 2020.

[11] Nazareth, "A relationship between the BFGS and conjugate gradient algorithms and its implications for new algorithms," SIAM Journal on Numerical Analysis, vol. 16, no. 5, pp. 794-800, 1979.

[12] C. Xue, T. Zhang, and D. Xiao, "Output-related and -unrelated fault monitoring with an improvement prototype knockoff filter and feature selection based on laplacian eigen maps and sparse regression," ACS Omega, vol. 6, no. 16, pp. 10828-10839, 2021.

[13] S. Yin, G. Wang, and H. Gao, "Data-driven process monitoring based on modified orthogonal projections to latent structures," IEEE Transactions on Control Systems Technology, vol. 24, no. 4, pp. 1480-1487, 2016.

[14] Z. Wu, S. Shen, H. Zhou, H. Li, C. Lu, and D. Zou, “An effective approach for the protection of user commodity viewing privacy in e-commerce website," Knowledge-Based Systems, vol. 220, Article ID 106952, 2021.

[15] Z. Wu, G. Li, S. Shen, X. Lian, E. Chen, and G. Xu, "Constructing dummy query sequences to protect location privacy and query privacy in location-based services," World Wide Web, vol. 24, no. 1, pp. 25-49, 2021.

[16] Z. Wu, S. Shen, X. Lian, X. Su, and E. Chen, “A dummy-based user privacy protection approach for text information retrieval," Knowledge-Based Systems, vol. 195, Article ID 105679, 2020.

[17] B. Wu, X. Y. Chen, Z. D. Wu, Z. Q. Zhao, Z. L. Mei, and C. C. Zhang, "Privacy-guarding optimal route finding with support for semantic search on encrypted graph in cloud computing scenario," Wireless Communications and Mobile Computing, vol. 2021, Article ID 6617959, 12 pages, 2021.

[18] X. C. Xie and S. Member, "An advanced PLS approach for key performance indicator- related prediction and diagnosis in case of outliers," IEEE Transactions on Industrial Electronics, vol. 63, no. 4, pp. 2587-2594, 2016.

[19] K. H. Yuan and P. M. Bentler, "Effect of outliers on estimators and tests in covariance structure analysis," The British Journal of Mathematical and Statistical Psychology, vol. 54, no. 1, pp. 161-175, 2001.

[20] C. C. Hsu, M. C. Chen, and L. S. Chen, "A novel process monitoring approach with dynamic independent component analysis," Control Engineering Practice, vol. 18, no. 3, pp. 242-253, 2010.

[21] H. Liu, S. Shah, and W. Jiang, "On-line outlier detection and data cleaning," Computers \& Chemical Engineering, vol. 28, no. 9, pp. 1635-1647, 2004.

[22] V. Hodge and J. Austin, "A survey of outlier detection methodologies," Artificial Intelligence Review, vol. 22, no. 2, pp. 85-126, 2004.

[23] L. H. Chiang, R. J. Pell, and M. B. Seasholtz, "Exploring process data with the use of robust outlier detection algorithms," Journal of Process Control, vol. 13, no. 5, pp. 437-449, 2003.
[24] P. J. Rousseeuw and C. Croux, "Alternatives to the median absolute deviation," Journal of the American Statistical, vol. 88, no. 424, pp. 1273-1283, 1993.

[25] S. Yin and G. Wang, "A modified partial robust M-regression to improve prediction performance for data with outliers," IEEE International Symposium on Industrial Electronics, pp. 1-6, 2013.

[26] S. Yin, S. X. Ding, A. Haghani, H. Hao, and P. Zhang, "A comparison study of basic data driven fault diagnosis and process monitoring methods on the benchmark tennessee eastman process," Journal of Process Control, vol. 22, no. 9, pp. 1567-1581, 2012.

[27] Y. Xu, S. Q. Shen, Y. L. He, and Q. X. Zhu, "A novel hybrid method integrating ICA-PCA with relevant vector machine for multivariate process monitoring," IEEE Transactions on Control Systems Technology, vol. 27, no. 4, pp. 1780-1787, 2018.

[28] W. Zou, Y. Xia, and H. Li, "Fault diagnosis of tennesseeeastman process using orthogonal incremental extreme learning machine based on driving amount," IEEE Transactions on Cybernetics, vol. 48, no. 12, pp. 3403-3410, 2018.

[29] J. J. Downs and E. F. Vogel, "A plant-wide industrial process control problem," Computers \& Chemical Engineering, vol. 17, no. 3, pp. 245-255, 1993.

[30] B. Jiang, "Maximized mutual information analysis based on stochastic representation for process monitoring," IEEE Transactions on Industrial Informatics, vol. 15, no. 3, 2019.

[31] A. Kocsor and L. Tóth, "Kernel based feature extraction with a speech technology application," IEEE Transactions on Signal Processing, vol. 52, no. 8, pp. 2250-2263, 2004.

[32] J. E. Garcia-Bracamonte, J. M. Ramirez-Cortes, J. de Jesus RangelMagdaleno, P. Gomez-Gil, H. Peregrina-Barreto, and V. AlarconAquino, "An approach on MCSA-based fault detection using independent component analysis and neural networks," IEEE Transactions on Instrumentation and Measurement, vol. 68, no. 5, pp. 1353-1361, 2019.

[33] J. M. Lee, S. J. Qin, and I. B. vLee, "Fault detection and diagnosis based on modified independent component analysis," AICHE Journal, vol. 52, no. 10, pp. 3501-3514, 2006.

[34] Y. Zhang and S. J. Qin, "Fault detection of nonlinear processes using multiway kernel independent analysis," Industrial \& Engineering Chemistry Research, vol. 46, no. 23, pp. 7780-7787, 2007.

[35] S. Rännar, F. Lindgren, P. Geladi, and S. Wold, "A pls kernel algorithm for data sets with many variables and fewer objects. Part 1: theory and algorithm," Journal of Chemometrics, vol. 8, no. 2, pp. 111-125, 1994.

[36] B. T. Le, D. Xiao, Y. C. Mao, D. K. He, J. L. Xu, and L. song, "Coal quality exploration technology based on an incremental multilayer extreme learning machine and remote sensing images," IEEE Transactions on Geoscience And Remote Sensing, vol. 57, pp. 4192-4201, 2019.

[37] G. C. Gajo, T. M. Assis, L. C. Assis, T. C. Ramalho, and E. F. F. Cunha, "Quantitative Structure-activity relationship studies for potential rho-associated protein kinase inhibitors," Journal of Chemistry, vol. 2016, Article ID 9198582, 12 pages, 2016. 